\title{
Collembolans accelerate the dispersal of antibiotic resistance genes in the soil ecosystem
}

\author{
Dong Zhu ${ }^{1,2}$, Hong-Tao Wang ${ }^{1,2}$, Fei Zheng ${ }^{1,2}$, Xiao-Ru Yang ${ }^{1}$, Peter Christie ${ }^{3}$, Yong-Guan Zhu ${ }^{1,2,4, *}$ \\ 1 Key Laboratory of Urban Environment and Health, Institute of Urban Environment, Chinese Academy of Sciences, \\ Xiamen 361021, China \\ 2 University of Chinese Academy of Sciences, $19 A$ Yuquan Road, Beijing 100049, China \\ 3 Agri-Environment Branch, Agri-Food and Biosciences Institute, Belfast BT9 5PX, United Kingdom \\ 4 State Key Laboratory of Urban and Regional Ecology, Research Center for Eco-Environmental Sciences, Chinese Academy of Sciences, \\ Beijing 100085, China
}

\section{ARTICLE I N F O}

Article history:

Received May 28, 2018

Revised September 10, 2018

Accepted September 11, 2018

\section{Keywords:}

Soil fauna

High-throughput qPCR

Microbial community

Mobile genetic elements

Lllumina sequencing

\begin{abstract}
A B S TR A C T
Soils have become an important sink for antibiotic resistance genes (ARGs). To better understand the impacts of ARGs on the soil ecosystem, the transport of ARGs is a basic question. So far, however, the role of soil animals in the dispersal of ARGs is not understood. Here, two treatments (without collembolans and with collembolans) were established, each treatment included unamended and manure-amended soil, and soil samples were collected at 14, 28 and 56 days after incubation. The effects of the collembolan Folsomia candida on dispersal of ARGs in the soil ecosystem were explored using high-throughput qPCR combined with Illumina sequencing. As the culture time increased, more shared ARGs and OTUs were detected between the unamended and manured soil, especially in the treatment with collembolans. Vancomycin, aminoglycoside and MLSB genes may have been more readily transported by the collembolan. On the 28th day after incubation, a high abundance of mobile genetic elements (MGEs) was found in the treatment with collembolans. These results clearly reveal that collembolans can accelerate the dispersal of ARGs in the soil ecosystem. Procrustes analysis and the Mantel test both indicate that soil bacterial communities were significantly correlated with ARG profiles. Furthermore, partial redundancy analysis indicates that soil bacterial communities can explain $41.28 \%$ of the variation in ARGs. These results suggest that the change of soil microbial community have an important contribution to the dispersal of ARGs by the collembolan.
\end{abstract}

(c) Higher Education Press 2019

\section{Introduction}

Antibiotic resistance is already considered to be a major threat to human health, and its emergence and dissemination have caused widespread public concern (Garofalo et al., 2007;

\footnotetext{
* Corresponding author

E-mail address: ygzhu@iue.ac.cn (Y.G. Zhu)
}

Organization, 2014). It is essential to recognize the factors affecting the transmission of ARGs in order to mitigate the problem of antibiotic resistance genes (ARGs) (Ashbolt et al., 2013; Bengtsson-Palme et al., 2018). Over the past few years, some factors (e.g., travel (Bengtsson-Palme et al., 2015), dust (Zhou et al., 2018), organic vegetables (Zhu et al., 2017a), application of manures and effluent discharge (Chen et al., 2016; Chu et al., 2018)) have been identified to be important in the spread of ARGs. However, our understanding of the 
spread of ARGs is still elusive (Bengtsson-Palme et al., 2018), especially in the soil ecosystem. Soils have become an important sink for ARGs because of the influence of human activities such as the application of manures and wastewater irrigation (Zhu et al., 2013). For instance, in a farmland of longterm applied sewage sludge, the abundance and diversity of ARGs were significantly increased in soils (Chen et al., 2016), and high abundance of ARGs was detected in the soil near the pig farm (Zhu et al., 2013). In addition, various animals inhabit in the soil (Thiele-Bruhn et al., 2012) and, in common with human travel (Bengtsson-Palme et al., 2015), we suspect that the activities of soil animals may also contribute to the spread of ARGs in the soil ecosystem. Because soil animals play an important role in material recycling, energy conversion and the transport of microbiota in the soil ecosystem (Hopkin, 1997; Fountain and Hopkin, 2005), and soil microbial community is commonly related to ARGs (Forsberg et al., 2014). Moreover, previous studies have also indicated that soil animal has an important contribution to the dispersal of microplastics (Maaß et al., 2017; Zhu et al., 2018b). However, the effects of soil animals on the dispersal of ARGs are unclear.

Collembolans are one of the most abundant groups of soil micro-arthropod and are widely distributed around the world (Hopkin, 1997; Rusek, 1998). Many species of collembolan have furcae or fork-like structures which extend their range of activities (Hopkin, 1997). The surfaces of collembolans are covered with waxy layers (Zhu et al., 2017b) and they can float on soil water and be carried by surface runwater. On the other hand, collembolan gut may be a "hot spot" for ARGs (Zhu et al., 2018a). A previous study has indicated that many ARGs have been detected in the collembolan gut microbiota (Zhu et al., 2018a). These suggested that the excretion of collembolan may have a contribution to the dispersal of ARGs. Collembolans are therefore excellent model animals in the study of the role of soil animals in the dispersal of ARGs in soils. The model collembolan Folsomia candida was used in our study. It is well-established that $F$. candida has been spread in various soils and plant pots all over the world as a result of human activities (Johnson and Wellington, 1983). Indeed, at some sites $F$. candida is the dominant animal species in the soil ecosystem (Fountain and Hopkin, 2005).

Here, the contribution of collembolan to the dispersal of ARGs was determined using the high throughput quantitative PCR in the soil ecosystem. Combined with 16S rRNA gene Illumina sequencing, the relationship between the dispersal of ARGs and bacterial community changes was also explored.

\section{Materials and methods}

\subsection{Test animal and soil}

Individuals (22-25 days old) of the collembolan Folsomia candida were used. The methods of animal culture and synchronization have been described in our previous study (Zhu et al., 2016). The test soil was collected from agricultural land at Ningbo, Zhejiang province, East China. The soil collected $\left(\mathrm{pH}\left(\mathrm{CaCl}_{2}\right) 4.76\right.$, total $\mathrm{C} 32.8 \mathrm{~g} \cdot \mathrm{kg}^{-1}$, total $\mathrm{N} 3.8$ $\mathrm{g} \cdot \mathrm{kg}^{-1}$ and CEC $13.86 \mathrm{cmol} \cdot \mathrm{kg}^{-1}$ ) was used as the unamended soil and was mixed with $2 \%$ pig manure (total $\mathrm{C}$ $218.9 \mathrm{mg} \cdot \mathrm{kg}^{-1}$, total $\mathrm{N} 28.9 \mathrm{mg} \cdot \mathrm{kg}^{-1}$, oxytetracycline 0.025 $\mathrm{mg} \cdot \mathrm{kg}^{-1}$, ofloxacin $0.021 \mathrm{mg} \cdot \mathrm{kg}^{-1}$ and sulfadimidine 0.004 $\mathrm{mg} \cdot \mathrm{kg}^{-1}$ ) to produce the ARG-enriched soil (manured soil).

\subsection{Experimental design}

Each test plastic container (height $8 \mathrm{~cm}$, inner diameter $6 \mathrm{~cm}$ ) was vertically divided into two halves with a plastic divider. Thirty grams (wet weight) of unamended soil were placed in one half of the container, $30 \mathrm{~g}$ of manured soil in the other half, and the plastic divider was then carefully removed. A total of 20 test containers were prepared, 10 of which received the collembolan and the other 10 remained animal-free as controls. Each container receiving collembolans received 50 synchronized $F$. candida (22-25 days old) individuals which were placed in the center of the container. Each container was sealed with a lid and the containers were incubated for 56 days at $20^{\circ} \mathrm{C}$ at $75 \%$ humidity. Sterile water was added twice weekly to maintain the moisture content. On the 14th day (three replicates per treatment), 28th day (three replicates per treatment) and 56th day (four replicates per treatment), randomly selected containers were destructively sampled. The unamended and manured soils were separated by reinserting the plastic divider in each container. The separate soils were carefully transferred to $100-\mathrm{mL}$ glass beakers, thoroughly mixed and stored at $-80^{\circ} \mathrm{C}$ prior to DNA extraction.

\subsection{Total soil DNA extraction and high-throughput quantitative PCR}

A FastDNA Spin Kit for soil (MP Biomedicals, Santa Ana, CA) was used to isolate soil DNA according to the manufacturer's instructions. In brief, $0.5 \mathrm{~g}$ soil (wet weight) was used to extract DNA and the isolated soil DNA was eluted with the $100 \mu \mathrm{L}$ DES solutions provided. The concentration and quality of the extracted DNA were checked and the DNA was then frozen at $-20^{\circ} \mathrm{C}$ prior to further analysis.

The DNA concentration in each soil sample was diluted to $50 \mathrm{ng} \cdot \mu \mathrm{L}^{-1}$ to detect $\mathrm{ARG}$ s using high-throughput quantitative PCR. A total of 296 primers (a 16S rRNA gene, 285 ARGs, 8 transposases, a class 1 intergron and a clinical class 1 integron) were included (Table S1). Three technical replicates per sample were used, and the genes were determined according to three positive replicates. The reaction system and conditions of the high-throughput qPCR and calculation method of ARG abundance were described in our previous study (Zhu et al., 2018a).

\subsection{Illumina sequencing and bioinformatics analysis}

The primers of $16 \mathrm{~S}$ rRNA gene V4-V5 region were used to amplify the extracted DNA samples in a $25 \mu \mathrm{L}$ reaction system equipped with unique barcodes to identify the samples. Further details of the PCR amplification were consistent with our previous study (Zhu et al., 2018a). The concentrations of 
PCR purified products were assayed using a Qubit 3.0 fluorimeter (Thermo Fisher Scientific, Waltham, MA), and products of equal quality were pooled to construct the sequencing library. The Illumina Hiseq2500 platform was used for high-throughput sequencing by Novogene, Beijing, China.

The sequences obtained were processed using Qiime 1.8.0 based on the online instructions (Caporaso et al., 2010b). Briefly, clean data were obtained by filtering primer sequences, low-quality reads and ambiguous nucleotides. UCLUST clustering was used to assign high quality sequences to the operational taxonomic units (OTUs) based on $3 \%$ sequence difference (Edgar, 2010). Only one sequence of OTUs was discarded. The GreenGenes 13.8 reference database was used to align the representative sequence of each OUT via PyNAST and to assign the taxonomy with RDP Classifier 2.2 (Caporaso et al., 2010a; McDonald et al., 2012). The phylogenetic tree was constructed using the Fast tree algorithm for further analysis (Price et al., 2010). The sequencing data are available at the NCBI Sequence Read Archive under the Bioproject PRJNA450153 with accession number SRP140545.

\subsection{Statistical processing}

Mean values of the data were calculated using Microsoft Excel 2013. Differences in ARGs between treatments at the 0.05 level were examined using one-way analysis of variance with the SPSS software package version 20.0. Histograms were drawn using OriginPro 9.1. The beta diversity of ARGs and bacterial communities was visualized by non-metric multidimensional scaling (NMDS) analysis using the vegan 2.4-3 (Oksanen et al., 2015) package of $R$ version 3.4.1 (RCoreTeam, 2017), and difference analysis was preformed using the Anosim test. A Venn diagram was produced using Venny 2.1 online. Redundancy analysis (RDA) and the Procrustes test were conducted to determine the relationships between soil ARGs and the bacterial community using the vegan 2.3-1 and labdsv 1.8-0 packages (Roberts, 2016).

\section{Results}

3.1 Collembolans alter the abundance and composition of ARGs

A total of 144 ARGs, 2 Integrase and 2 Transposase were detected in our all samples, including major classes with antibiotic resistance (e.g. aminoglycoside, beta-lactams, chloramphenicol, macrolide-lincosamide-streptogramin B (MLSB), multidrug, sulfonamide, tetracycline, vancomycin and others). At 14 days, the ARG number (treatment without collembolans: 2.2 fold and treatment with collembolans: 1.6 fold) and abundance (treatment without collembolans: 2.9 fold and treatment with collembolans: 3.0 fold) in the manured soil were significantly higher than in the unamended soil $(P<0.05$, Fig. 1). At 28 days there were no significant differences in the number and abundance of ARGs between the unamended and manured soil in the presence of the collembolans $(P>0.05$, Fig. 1). However, in the treatment without collembolans, the numbers and abundance of ARGs in the manured soil were still significantly higher than those of the unamended soil, by $145 \%$ and $407 \%$, respectively $(P<0.05$, Fig. 1$)$. On day 28 the number and abundance of ARGs in the unamended soil of the treatment with collembolans were 102 and $614 \%$ higher, respectively $(P<0.05)$ than in the treatment without collembolans (Fig. 1). Moreover, at 28 days the number and abundance of vancomycin, aminoglycoside and MLSB genes were higher in the unamended soil of the
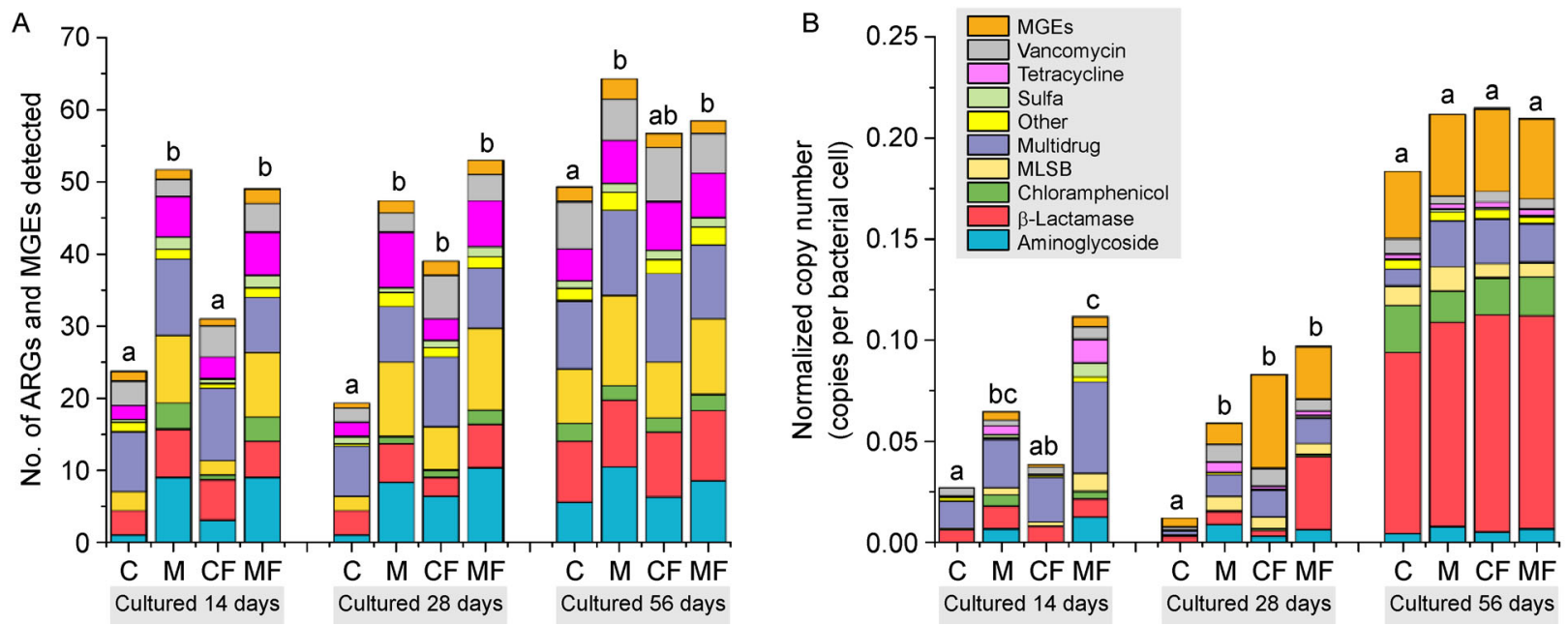

Fig. 1 (A) Detected number and (B) normalized copy number of ARGs and MGEs (mean, $n \geqslant 3$ ) in the soil microbial community from different culture stages $(14,28$ and 56 days). $C$, the unamended soil in the treatment without collembolans; M, the manured soil in the treatment without collembolans; $\mathrm{CF}$, the unamended soil in the treatment with collembolans; MF, the manured soil in the treatment with collembolans. ARGs were classified based on the class of antibiotic they resisted. The lowercase indicated significant difference between different treatments of the same stage at 0.05 level (LSD test). 
treatment with collembolans than those in the treatment without collembolans $(P<0.05$, Fig. 1). After 28 days the abundance of MGEs in the unamended soil of the treatment with collembolans was 10 -folds higher than that in the treatment without collembolans $(P<0.05$, Fig. 1B). After 56 days the abundance of ARGs was similar in all treatments, but in the treatment without collembolans the number of ARGs in the manured soil was still $30 \%$ higher $(P<0.05)$ than in the unamended soil. The NMDS analysis of ARG composition also shows that all samples clusters with increasing culture time (Fig. S1). At 14 days the compositions of ARGs from differently treated samples were clustered by manure treatment (Anosim, $P=0.003$ ). The unamended soil samples of the treatment without collembolans were clearly separated from the other samples (Anosim, $P=0.03$ ) on the 28th day.

3.2 The relationship between the dispersal of ARGs and bacterial community changes

We used the Mantel test and Procrustes analysis to explore the relationship between the bacterial community and ARG profile. The results all indicate a significant correlation between ARG profiles and bacterial communities $(P<0.05$, Fig. 2A). We further investigated the bacterial communities and variation in ARGs using partial redundancy analysis (pRDA) which confirms that soil bacterial communities can explain $41.28 \%$ of the ARG variation (Fig. $2 B$ ).

\subsection{Effects of collembolans on bacterial community characteristics}

After 14 and 28 days the bacterial community in the presence of the collembolan differed from the control at phylum and family levels (Fig. S2) and NMDS analysis also show a significant change in the bacterial community between different treatments (Anosim, $P<0.05$, Fig. 4). In the presence of the collembolan, the four predominant phyla (Actinobacteria, Firmicutes, Proteobacteria and Bacteroidetes) were not significantly different between the unamended and manured soil. However, without collembolanss, on days 14 and 28 the bacterial community of the unamended soil was significantly different from that of the manured soil (Anosim, $P<0.05$, Fig. S2 and Fig. 4). In the NMDS analysis we found a high heterogeneity of microbial communities in the unamended soil of the treatment with collembolans. Overall, the Venn diagram also shows that more shared OTUs were found between the unamended soil and manured soil with increasing culture time (Fig. 3B).

\section{Discussion}

Our study indicates that the ARGs can spread from one soil to another and the collembolan can speed up the dispersal process. For the contribution of the collembolan to the dispersal of the ARGs, we summarized five potential mechanisms. First, the collembolan can promote microbial transport in the soil ecosystem (Hopkin, 1997; Fountain and Hopkin, 2005), and the microorganisms may be hosts of the ARGs. Second, our previous study indicated that the collembolan gut can become enriched in ARGs (Zhu et al., 2018a) and thus the excreta of the collembolan may represent a pathway for the transport of ARGs. Third, the collembolan may prefer to ingest certain microorganisms (Lee and Widden, 1996; Hopkin, 1997; Nakamori and Suzuki, 2005) and this may alter the soil microbial community. Numerous studies have shown that changes in the microbial community make an important contribution to ARG shift (Forsberg et al., 2014; Zhu et al., 2018a). Four, the activity of the collembolan can alter soil micro-structure (Davidson and Grieve, 2006; Siddiky et al., 2012) and this may contribute to the spread of the ARGs. Some regions of the soil micro-structure may be
A

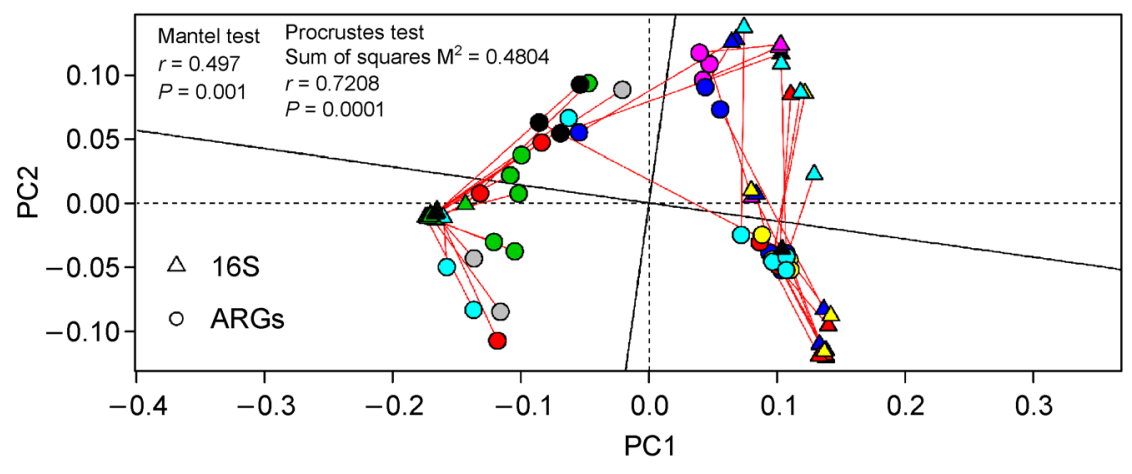

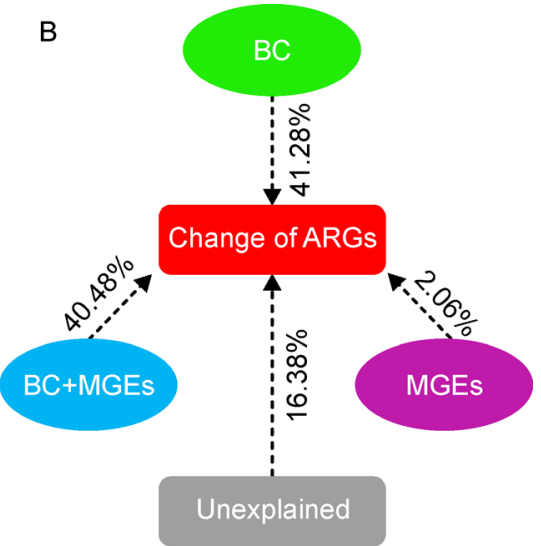

Fig. 2 (A) Procrustes test revealing the significant correlation between ARGs profile and soil bacterial taxa composition (16S rRNA gene OTU data) on the basis of Bray - Curtis dissimilarity metrics (sum of squares $\mathrm{M}^{2}=0.4804, r=0.7208, P=0.0001,9999$ permutations). The triangles represented 16S rRNA gene OTU data of soil microbial composition, the circles indicated different ARGs, and different colors represented different soil samples. The Mantel test was also preformed to confirm the relationship between ARGs and soil bacterial communities based on Bray - Curtis distance. (B) Partial redundancy analysis conformed the contribution of bacterial community (BC) and MGEs to variations of ARGs. 
A
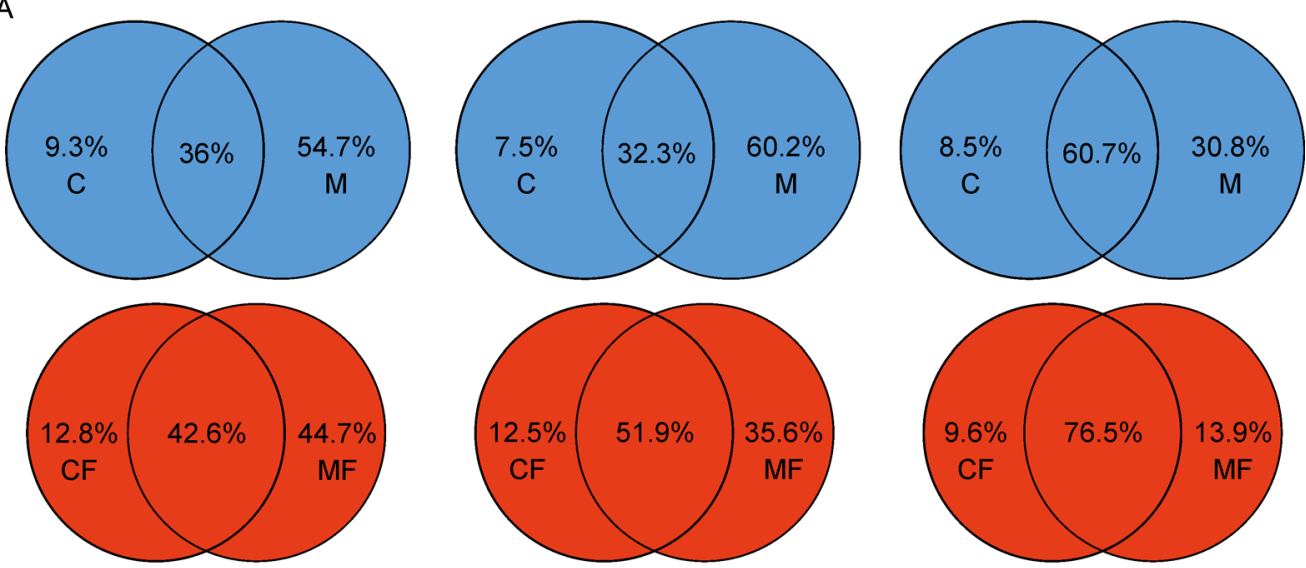

Cultured 14 days

Cultured 28 days

Cultured 56 days

B
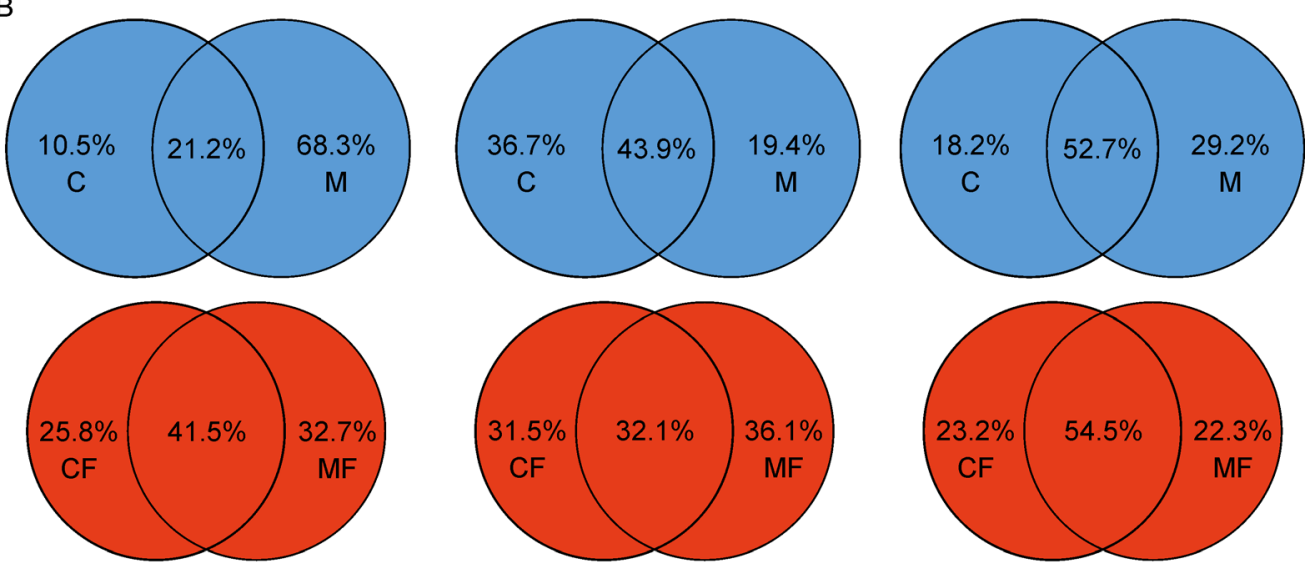

Cultured 28 days

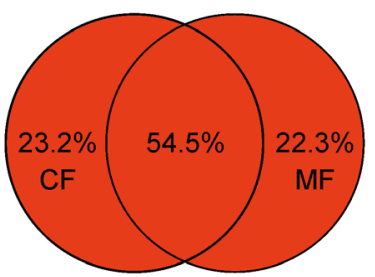

Cultured 14 days

Cultured 56 days

Fig. 3 Venn diagrams showing the percentage of shared ARGs (A) and OTUs (B) between different treatments of the same culture stage. C, the unamended soil in the treatment without collembolans; $M$, the manured soil in the treatment without collembolans; $C F$, the unamended soil in the treatment with collembolans; MF, the manured soil in the treatment with collembolans.

hotspots for microorganisms (Paradelo and Barral, 2009). Finally, since there are so many collembolan individuals concentrated in so little space (height $8 \mathrm{~cm}$, inner diameter 6 $\mathrm{cm}$ ), the activity of the collembolan might mix much of the two material. The Venn diagram shows that the presence of the collembolan leads to more shared ARGs between the unamended and the manured soil compared to the absence of the animals (Fig. 3). This also supports our conclusion that the collembolan can accelerate the dispersal of ARGs. The Venn diagram also indicates that the number of shared OTUs would increase with increasing culture time, especially in the presence of the collembolan, and this provides direct evidence of the spread of ARGs from one soil to another.

More vancomycin, aminoglycoside and MLSB genes were detected in the unamended soil of the treatment with collembolans compared to the treatment without collembo- lans, suggesting that these ARGs may be more readily transported by the collembolan in the soil ecosystem. Moreover, these ARGs are usually found in ARG-contaminated soils (Zhu et al., 2013; Chen et al., 2016) and their resistance antibiotics are commonly used in veterinary and human medicine (Duranti et al., 2017). Collembolans may therefore make an important contribution to the soil dispersal of vancomycin, aminoglycoside and MLSB genes in the field. It is well established that MGEs play an important role in the exchange of ARGs between microorganisms (Gaze et al., 2011). In the present study, high abundances of MGEs were found in the presence of the collembolan and this suggests the occurrence of the horizontal transfer of large numbers of ARGs. Previous studies also show that MGEs make an important contribution to the proliferation of ARGs (Forsberg et al., 2014; Chen et al., 2016). These results indicate that the 


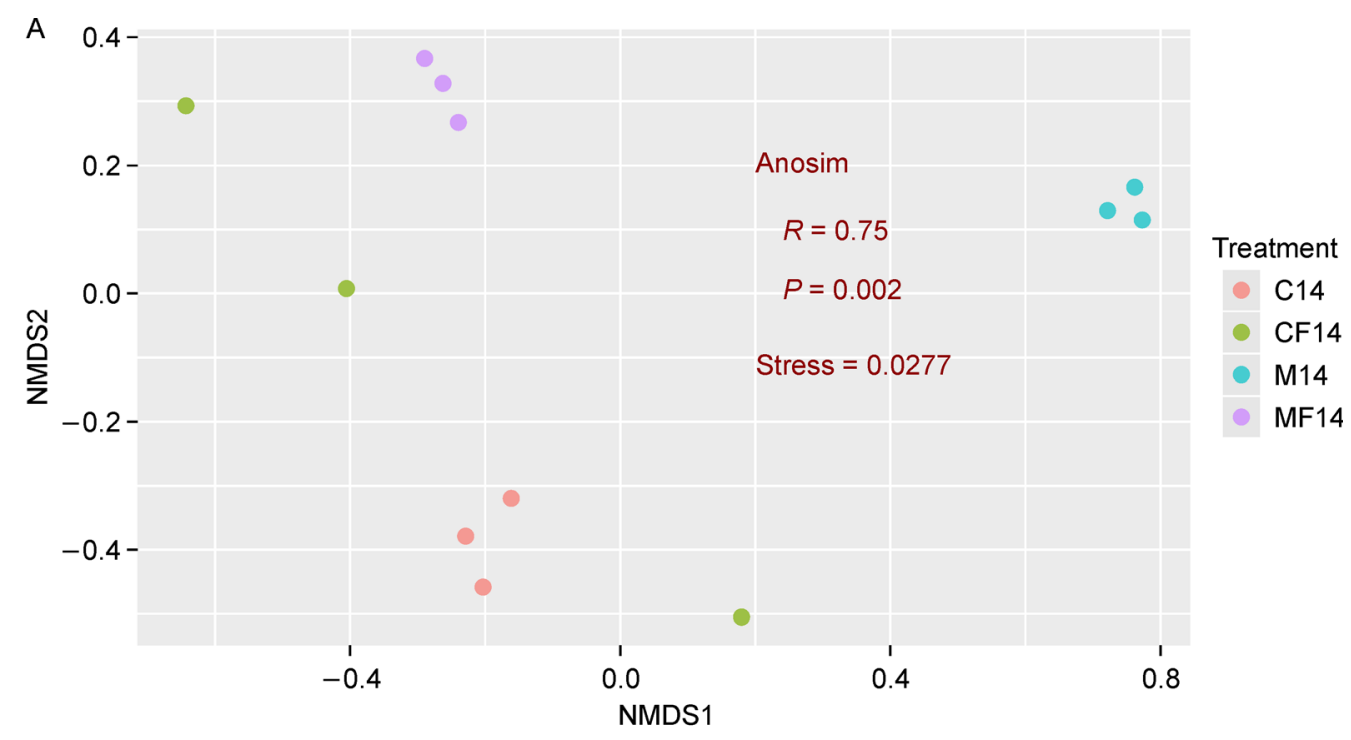

B
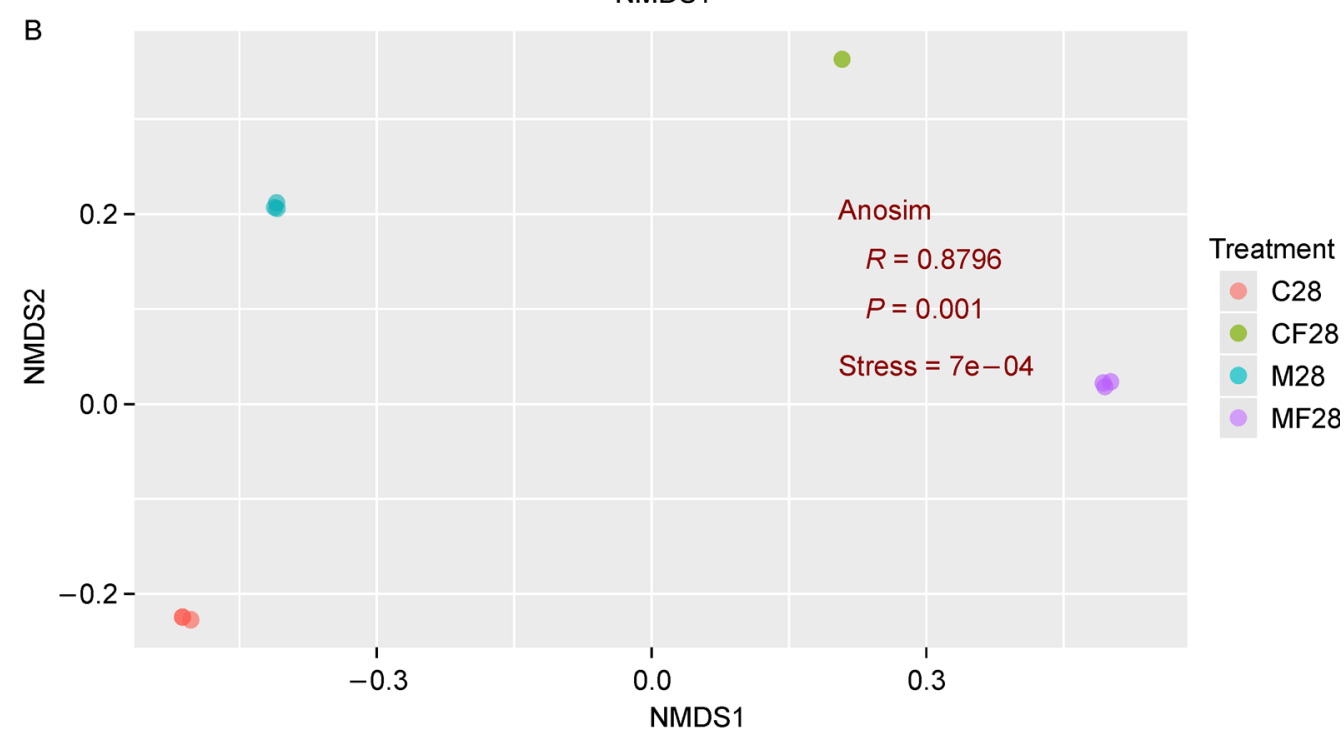

C 0.10

NMDS1

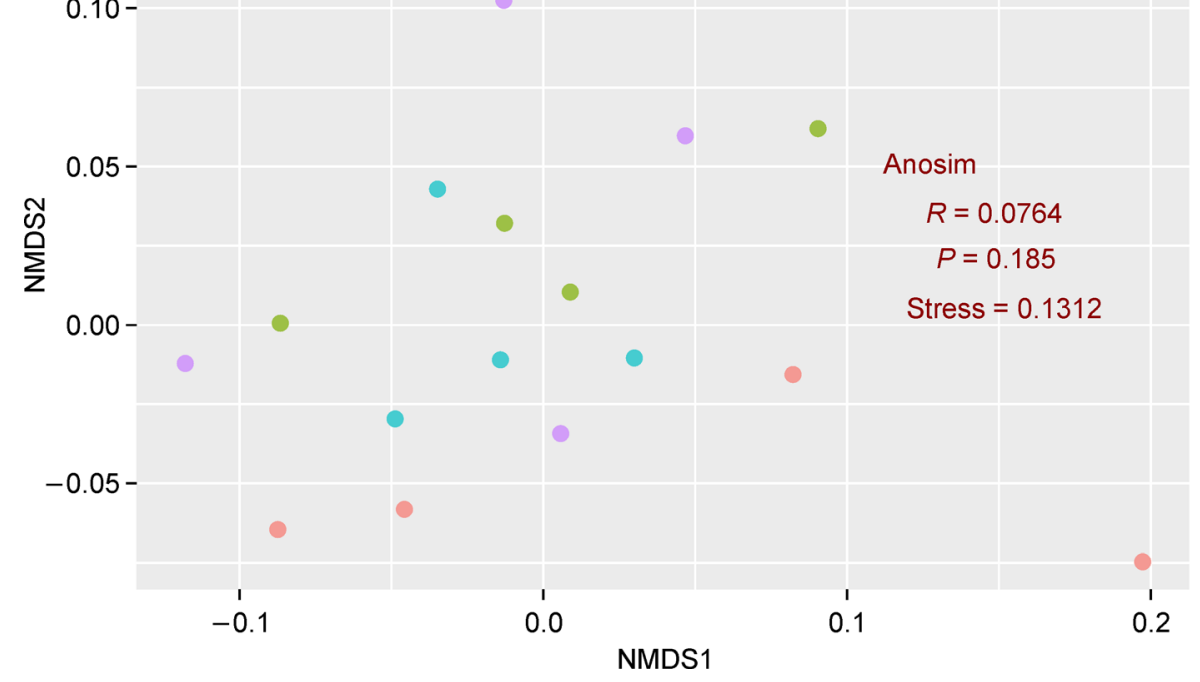

Fig. 4 Non-metric Multidimensional scaling (NMDS) analysis depicting the overall distribution pattern of soil bacterial communities in different stages (14 (A), 28 (B) and 56 (C) days) based on OTU table of the Bray-Curtis distance. The Anosim test was adopted to compare the difference of microbial community in different treatments at 0.05 level. 
collembolan may be able to increase the abundance of MGEs to affect the dispersal of ARGs, and the view also supported by the pRDA (Fig. 2B) in which MGEs explained $2.06 \%$ of the variation in ARGs.

Results of the Mantel test and Procrustes analysis all suggested that changes in soil bacterial communities may make an important contribution to the shift in ARG structure, which was also supported by the result of pRDA. Numerous previous studies have also shown that the microbial community is a major factor influencing ARG shift (Forsberg et al., 2014; Chen et al., 2016; Zhu et al., 2018a). At the same time, our results indicate that the presence of collembolans might significantly alter the soil bacterial community. This may be due to the ingestion and transport of microorganisms by the collembolan (Hopkin, 1997; Fountain and Hopkin, 2005). In the present study, the collembolans can promote communication between the bacterial communities of different soils. The result of the NMDS analysis also suggests that bacterial communities in the unamended soil might be changing greatly due to the activity of collembolans. Therefore, these results suggest that the collembolan can accelerate the dispersal of ARGs by altering the soil microbial community.

\section{Conclusion}

In summary, the role of soil collembolans in the dispersal of ARGs was investigated in the present study using HT-qPCR combined with high-throughput sequencing. The collembolan did indeed accelerate the dispersal of ARGs in the soil ecosystem. The presence of the collembolan leads to more shared ARGs which were detected between the unamended soil and the manured soil, as the culture time increased. The vancomycin, aminoglycoside and MLSB genes may be more readily transported by the collembolan and the animals may promote the dispersal of ARGs by increasing the abundance of MGEs and altering the bacterial community. These results will contribute to elevating our knowledge of the role of soil animals in the dispersal of ARGs in the environment.

\section{Acknowledgments}

This work was funded by the National Natural Science Foundation of China (41571130063), the Strategic Priority Research Program of the Chinese Academy of Sciences (XDB15020302 and XDB15020402) and the National Key Research and Development Program of China-International collaborative project from Ministry of Science and Technology (Grant No. 2017YFE0107300).

\section{Electronic supplementary material}

Supplementary material is available in the online version of this article at http://dx.doi.org/10.1007/s42832-019-0002-1 and is accessible for authorized users.

\section{References}

Ashbolt, N.J., Amézquita, A., Backhaus, T., Borriello, P., Brandt, K.K., Collignon, P., Coors, A., Finley, R., Gaze, W.H., Heberer, T., Lawrence, J.R., Larsson, D.G., McEwen, S.A., Ryan, J.J., Schönfeld, J., Silley, P., Snape, J.R., Van den Eede, C., Topp, E., 2013. Human Health Risk Assessment (HHRA) for environmental development and transfer of antibiotic resistance. Environmental Health Perspectives 121, 993-1001.

Bengtsson-Palme, J., Angelin, M., Huss, M., Kjellqvist, S., Kristiansson, E., Palmgren, H., Larsson, D.G., Johansson, A., 2015. The human gut microbiome as a transporter of antibiotic resistance genes between continents. Antimicrobial Agents and Chemotherapy $59,6551-6560$.

Bengtsson-Palme, J., Kristiansson, E., Larsson, D.G.J., 2018. Environmental factors influencing the development and spread of antibiotic resistance. FEMS Microbiology Reviews 42, 68-80.

Caporaso, J.G., Bittinger, K., Bushman, F.D., DeSantis, T.Z., Andersen, G.L., Knight, R., 2010a. PyNAST: a flexible tool for aligning sequences to a template alignment. Bioinformatics (Oxford, England) 26, 266-267.

Caporaso, J.G., Kuczynski, J., Stombaugh, J., Bittinger, K., Bushman, F.D., Costello, E.K., Fierer, N., Peña, A.G., Goodrich, J.K., Gordon, J.I., Huttley, G.A., Kelley, S.T., Knights, D., Koenig, J.E., Ley, R.E., Lozupone, C.A., McDonald, D., Muegge, B.D., Pirrung, M., Reeder, J., Sevinsky, J.R., Turnbaugh, P.J., Walters, W.A., Widmann, J., Yatsunenko, T., Zaneveld, J., Knight, R., 2010b. QIIME allows analysis of high-throughput community sequencing data. Nature Methods 7, 335-336.

Chen, Q., An, X., Li, H., Su, J., Ma, Y., Zhu, Y.G., 2016. Long-term field application of sewage sludge increases the abundance of antibiotic resistance genes in soil. Environment International 9293, 1-10.

Chu, B.T.T., Petrovich, M.L., Chaudhary, A., Wright, D., Murphy, B., Wells, G., Poretsky, R., 2018. Metagenomics reveals the impact of wastewater treatment plants on the dispersal of microorganisms and genes in aquatic sediments. Applied and Environmental Microbiology 84, e02168-e17.

Davidson, D.A., Grieve, I.C., 2006. The influence of soil fauna on soil structural attributes under a limed and untreated upland grassland. Land Degradation \& Development 17, 393-400.

Duranti, S., Lugli, G.A., Mancabelli, L., Turroni, F., Milani, C., Mangifesta, M., Ferrario, C., Anzalone, R., Viappiani, A., van Sinderen, D., Ventura, M., 2017. Prevalence of antibiotic resistance genes among human gut-derived bifidobacteria. Applied and Environmental Microbiology 83, e02894-e16.

Edgar, R.C., 2010. Search and clustering orders of magnitude faster than BLAST. Bioinformatics (Oxford, England) 26, 2460-2461.

Forsberg, K.J., Patel, S., Gibson, M.K., Lauber, C.L., Knight, R., Fierer, N., Dantas, G., 2014. Bacterial phylogeny structures soil resistomes across habitats. Nature 509, 612-616.

Fountain, M.T., Hopkin, S.P., 2005. Folsomia candida (Collembola): a "standard" soil arthropod. Annual Review of Entomology 50, 201, 
201-222.

Garofalo, C., Vignaroli, C., Zandri, G., Aquilanti, L., Bordoni, D., Osimani, A., Clementi, F., Biavasco, F., 2007. Direct detection of antibiotic resistance genes in specimens of chicken and pork meat. International Journal of Food Microbiology 113, 75-83.

Gaze, W.H., Zhang, L., Abdouslam, N.A., Hawkey, P.M., Calvo-Bado, L., Royle, J., Brown, H., Davis, S., Kay, P., Boxall, A.B.A., Wellington, E.M., 2011. Impacts of anthropogenic activity on the ecology of class 1 integrons and integron-associated genes in the environment. ISME Journal 5, 1253-1261.

Hopkin, S.P., 1997. Biology of the Springtails (Insecta: Collembola). Oxford University Press.

Johnson, D.L., Wellington, W.G., 1983. Dispersal of the collembolan Folsomia candida Willem, as a function of age. Canadian Journal of Zoology 61, 2535-2538.

Lee, Q., Widden, P., 1996. Folsomia candida, a "fungivorous" collembolan, feeds preferentially on nematodes rather than soil fungi. Soil Biology \& Biochemistry 28, 689-690.

Maaß, S., Daphi, D., Lehmann, A., Rillig, M.C., 2017. Transport of microplastics by two collembolan species. Environ Pollut 225, 456-459.

McDonald, D., Price, M.N., Goodrich, J., Nawrocki, E.P., DeSantis, T. Z., Probst, A., Andersen, G.L., Knight, R., Hugenholtz, P., 2012. An improved Greengenes taxonomy with explicit ranks for ecological and evolutionary analyses of bacteria and archaea. ISME Journal 6, 610-618.

Nakamori, T., Suzuki, A., 2005. Spore-breaking capabilities of collembolans and their feeding habitat within sporocarps. Pedobiologia 49, 261-267.

Oksanen, J., Blanchet, F.G., Kindt, R., Legendre, P., Minchin, P.R., O'Hara, R.B., Simpson, G.L., Solymos, P., Stevens, M.H.H., Wagner, H., 2015. vegan: Community Ecology Package. R package version 2.3-1. Lorenzo Cachón Rodríguez 48, págs.103-132.

Organization, W.H., 2014. Antimicrobial resistance: global report on surveillance. Australasian Medical Journal 7, 237.

Paradelo, R., Barral, M.T., 2009. Effect of moisture and disaggregation on the microbial activity of soil. Soil \& Tillage Research 104, 317 319.

Price, M.N., Dehal, P.S., Arkin, A.P., 2010. FastTree 2-approximately maximum-likelihood trees for large alignments. PLoS One 5, e9490.
RCoreTeam, 2017. A Language and Environment for Statistical Computing; R Foundation for Statistical Computing: Vienna, Austria; $h t t p: / / w w w . R-p r o j e c t . o r g$.

Roberts, D.W., 2016. labdsv: Ordination and Multivariate Analysis for Ecology; http://th.archive.ubuntu.com/cran/web/packages/labdsv/.

Rusek, J., 1998. Biodiversity of Collembola and their functional role in the ecosystem. Biodiversity and Conservation 7, 1207-1219.

Siddiky, M.R.K., Schaller, J., Caruso, T., Rillig, M.C., 2012. Arbuscular mycorrhizal fungi and Collembola non-additively increase soil aggregation. Soil Biology \& Biochemistry 47, 93-99.

Thiele-Bruhn, S., Bloem, J., de Vries, F.T., Kalbitz, K., Wagg, C., 2012. Linking soil biodiversity and agricultural soil management. Current Opinion in Environmental Sustainability 4, 523-528.

Zhou, H., Wang, X., Li, Z., Kuang, Y., Mao, D., Luo, Y., 2018. Occurrence and distribution of urban dust-associated bacterial antibiotic resistance in northern China. Environmental Science \& Technology Letters 5, 50-55.

Zhu, B., Chen, Q., Chen, S., Zhu, Y.G., 2017a. Does organically produced lettuce harbor higher abundance of antibiotic resistance genes than conventionally produced? Environment International 98, 152-159.

Zhu, D., An, X.L., Chen, Q.L., Yang, X.R., Christie, P., Ke, X., Wu, L.H., Zhu, Y.G., 2018a. Antibiotics disturb the microbiome and increase the incidence of resistance genes in the gut of a common soil collembolan. Environmental Science \& Technology 52, 30813090.

Zhu, D., Bi, Q.F., Xiang, Q., Chen, Q.L., Christie, P., Ke, X., Wu, L.H., Zhu, Y.G., 2018b. Trophic predator-prey relationships promote transport of microplastics compared with the single Hypoaspis aculeifer and Folsomia candida. Environ Pollut 235, 150-154.

Zhu, D., Ke, X., Wu, L., Christie, P., Luo, Y., 2016. Biological transfer of dietary cadmium in relation to nitrogen transfer and ${ }^{15} \mathrm{~N}$ fractionation in a soil collembolan-predatory mite food chain. Soil Biology \& Biochemistry 101, 207-216.

Zhu, P., Kong, T., Tang, X., Wang, L., 2017b. Well-defined porous membranes for robust omniphobic surfaces via microfluidic emulsion templating. Nature Communications 8, 15823.

Zhu, Y.G., Johnson, T.A., Su, J.Q., Qiao, M., Guo, G.X., Stedtfeld, R. D., Hashsham, S.A., Tiedje, J.M., 2013. Diverse and abundant antibiotic resistance genes in Chinese swine farms. Proceedings of the National Academy of Sciences of the United States of America 110, 3435-3440. 